PETER CLEAVE

\title{
Two Suns? The Algorithmic State: History and Theory
}

What follows is a history of cultural blindness. We might need to consider our historiography, the culture we find in the past, if you like, and the need to now reconstitute and re-frame that sense of history making the development of robotics and machine learning more central. Over in a usually forgotten corner there is a history of machines that may throw light on where we find ourselves today and in this section there is a consideration of that history, a story about robotics and theorists in this area unheard and unseen in many respects.

We might also, with a nod to Mannheim and others look at ideology and we might look again at Foucault's idea of an episteme. In whatever discursive route is taken there is usually a sense that men run the machines. In recent times there is something or a catharsis or at least a set of realisations about people and machines and such 
terms as cobotics where the two work together have been discussed.

An example of cobotics might be that if we go back to the book, The Warehouse, there the worker goes from stack to stack picking and packing sometimes using belts and hooks to reach high on a stack. Now in Amazon Fulfillment Centres, the stacks move to the workers and lights in the bins on the stacks indicate where to pick, messages are sent between the vests workers wear and other points in the warehouse and generally the worker is stationary now while robots do a great deal of the moving around. In a few short years the cobotic situation has strengthened and developed considerably so that instead of people coming to the machines the machines come to the people.

In what follows an attempt at explanation of this history that we did not notice is made. What should we be noticing now? Such a question might be impossible to answer but we may have a crack at it as we go. What are the facts in that history, what happened when and where? And what are the myths in that history, what is the shorthand and what are the extended treatments?

We could go back to a history of illusion as Vaucansun (1709-1782), with a mix of science and fairground schlock, gave us the mechanical chess player as well as his shitting duck, all in a spirit of curiosity, all too in a mix of the friendly and the cute with the machine. We could go back to the history 
of dolls and to dolls in cultures around the world with Gaby Wood's Living Dolls in mind to karetao in the New Zealand Maori case and look at the literature there to see if there are significant cultural and epistemological differences that might themselves shed light on the current situation and the use of the term 'cobotics' with regard, say, to the person and their pet computer, robot, scout, car or any other machine that they operate at a distance sometimes even only to say 'Stop' and 'Go' or 'Its time to retire for repair or replacement'.

We could walk a path or two trying to follow theorists of society covering the period since Vaucansun. Foucault talked about the emergence of man as a subject. And yet over the same period we can see the emergence of the machine. We never saw the machine as the central focus, the machine as subject.

In the writings of HG Wells and others the machines are imagined to be in competition with humans. $\mathrm{H}$ G Wells might himself be the 'go to' person for the imagining of the machine. But there is a need to consider Huxley and other writers as its not all about the emergence of the machine, its also to do with the focus on pleasant diversion facilitated by various technologies in the face of or in the presence of the machine taking that word to mean developments in AI as well as the usual connotations. And then we might think about Orwell and, perhaps, throw in a pinch of Phillip K Dick. We 
might call this Science Fiction or we might see this literature as more central to our experience just as we might see a more central role for Machine Learning discussed below.

We could talk about the industrial age and the information age and suggest that we might have missed the essential industry of the industrial age which became the workings of the algorithm, of a system to take a formula of analysis, repeat it and improve it, then proceed into extrapolation, to learning. As O'Neill and Sauter point out in the discussion in Seven and elsewhere in the Two Suns? series the algorithm can be simple, it can be deceptive but it can also be extremely complex with high capacity for learning. How did this come to be? We might question the idea of revolution as in the sense of the industrial revolution. In which direction was there a turning? Toward the freeing of people from repetitive work or of freeing machines to learn, albeit at first in a stumbling, barely recognised way as the history sketched below shows. To understand what is happening with Big Tech and what I have called the Algorithmic State we might do such historical research on such things as robotics as we find a way forward to talk about cobotics, governance and the sovereignty of systems.

We seem to have set of silences, of historical glitches, of missed gear shifts where almost out of nowhere comes a Bayes or a Babbage. And then another gap and then its Learning 
Machines/Machine Learning. This is considered below in terms of what is known as 'the AI winter' and the way we bracket out developments in this area.

In the broad picture we really need to go back to a history of government or governance. We are used to a history of governance by government but now we might speak about other forms of governance involving algorithms, about cobotics and the use of systems. The discussion of techno feudalism in the previous section of this series might inform a consideration of governance but it might also miss the central matters of the data and the algorithm, the robot and then the cobot, the leaders who were only as good as their data could become, as their data could make them.

The history outlined below happened over a period that saw the Romantic Poets, almost as though the emergence of machine culture called forth an interest in self and inner landscapes. Over a period that saw the development of the great democracies in France, the USA and in England we have also seen the development in California and elsewhere of what commentators have called techno feudalism and what has been called in this series the Algorithmic State.

Are our historical preoccupations to do with an unconscious rejection of the learning machine and its antecedents? Are our attentions to the arts and politics a set of unconsciously willed distractions, 
ways of missing the point and escaping the plot? So much of our politics and arts are to do with egalitarian ideas and now we find a gap of outrageous proportions between Bezos, the richest man in the world, and Gates, Musk, Zuckerberg and a few others and the person on the Clapham omnibus. How, and to the point of this part of the Two Suns? series when did this happen?

Do we have theory for algorithm and resource over time? One thinks of fisheries, quota and algorithms, of data and quota. In a paper of 1999 the present author went over this general position.

But something else was happening from Vaucansun in the time of Voltaire with the first robots to a situation of Machine Learning where robots teach themselves. And we missed it. We could go back a long way and take several perspectives. One perspective might be to look at the division of space and extant interests or lack thereof. In the 1880s there was the partition of Africa but this, at the Berlin conference of 1878, might not have been given great prominence in Europe. In 1949 there was the partition of India. Again that happened in a place obscure to many observers in Europe and the Americas and elsewhere.

The discussion of the algorithmic state follows a similar pattern. An obscure area like space can be characterised as a shell belonging to Musk or Bezos and then turn out to be significant in communications. Developments in AI or machine 
learning happen in obscurity and then turn out to be central. We could say the history of the latter is obscure pretty well up to the latter years of the last century and then becomes increasingly important. Even in house though amongst those working in or close to the field there are silences as with what is known as 'the AI winter' in part of the last half of last century.

This leaves us with a historiography where almost out of nowhere in the 1990s there is a breakout and this is the point, the moment, perhaps when the algorithmic state starts to emerge, to emerge out of the chrysalis, as it were, from the AI laboratory to the real world. Then, as machine learning develops there is a process of leaps and bounds. There is also a moment in 2016-7 in the case of the Cambridge Analytica matter where the wide world wakes up, as it were, to the idea that data might be abused.

And its all to do, practically speaking, with the way robots work in Amazon's fulfilment centres, to drone delivery through Prime Air, the way your computer tracks your interests or the ways in which the movements of you and your dog are followed in the neighbourhood mesh. How did all this happen? And when?

Somehow we don't bother to explain this, we simply accept and move on. But there is a general thing about the sky and close or inner space that has happened over the last half century that was not there before. There is an aspect to the 
neighbourhood, to the home, to the screen that was not there before, there has been a shaping of experience.

Now might be a good time to walk through the history slowly and carefully to see how we arrived here. What follows is a slow and steady year by year recounting rather than a display of historical erudition. The intention is to set out a platform of facts over time using standard references. Having said that we might also start at the end or one of the ends. Wikipedia provides an excellent introduction to machine learning,

The term machine learning was coined in 1959 by Arthur Samuel, an American IBMer and pioneer in the field of computer gaming and artificial intelligence. A representative book of the machine learning research during the 1960s was the Nilsson's book on Learning Machines, dealing mostly with machine learning for pattern classification. Interest related to pattern recognition continued into the 1970s, as described by Duda and Hart in 1973. In 1981 a report was given on using teaching strategies so that a neural network learns to recognize 40 characters (26 letters, 10 digits, and 4 special symbols) from a computer terminal.

Tom M. Mitchell provided a widely quoted, more formal definition of the algorithms studied in the machine learning field: "A computer program is said to learn from experience $\mathrm{E}$ with respect to some 
class of tasks $\mathrm{T}$ and performance measure $\mathrm{P}$ if its performance at tasks in $\mathrm{T}$, as measured by $\mathrm{P}$, improves with experience E." This definition of the tasks in which machine learning is concerned offers a fundamentally operational definition rather than defining the field in cognitive terms. This follows Alan Turing's proposal in his paper "Computing Machinery and Intelligence", in which the question "Can machines think?" is replaced with the question "Can machines do what we (as thinking entities) can do?".

Modern day machine learning has two objectives, one is to classify data based on models which have been developed, the other purpose is to make predictions for future outcomes based on these models. A hypothetical algorithm specific to classifying data may use computer vision of moles coupled with supervised learning in order to train it to classify the cancerous moles. Whereas, a machine learning algorithm for stock trading may inform the trader of future potential predictions.

The entry for Machine Learning in Wikipedia above begins in the 1950s and talks about statistical methods being discovered and refined at that time. At the same time simple algorithms are used in 'pioneering machine learning research'. One curious feature is that notwithstanding the figures mentioned above and their times there is a return to the eighteenth century and the mathematics of Thomas Bayes (1701-1761). 
To look at the prehistory of Machine Learning, going back to 1763 we can see the underpinnings of Bayes' Theorem. Thomas Bayes's work An Essay towards solving a Problem in the Doctrine of Chances is published two years after his death, having been amended and edited by a friend of Bayes, Richard Price. The essay presents work which underpins Bayes theorem.

Then in 1805, in the time of Napoleon Bonaparte (1769-1821) there is the business of the Least Square. Adrien-Marie Legendre describes the 'méthode des moindres carrés', known in English as the least squares method. The least squares method is used widely in data fitting. In 1812 there is the development of Bayes' Theorem. Pierre-Simon Laplace publishes Théorie Analytique des Probabilités, in which he expands upon the work of Bayes and defines what is now known as Bayes' Theorem.

We could go back to Babbage (1791-1871), his Difference Engine and his Analytical Engine which puts us, going back, toward the Enlightenment, to Vaucansun (1709 -1782) and Voltaire (1694-1778) but also, going the other way in time, toward Darwin (1809-1882). If the latter thought much of machines little was the telling. It is interesting though to think of the reception to Darwin's ideas. The AI winter of the 1970s discussed later might be compared to the treatment of Darwin's work in the early twentieth century as recounted on Wikipedia: 
Around the beginning of the 20th century, a period that has been called "the eclipse of Darwinism", scientists proposed various alternative evolutionary mechanisms, which eventually proved untenable. Ronald Fisher, an English statistician, finally united Mendelian genetics with natural selection, in the period between 1918 and his 1930 book The Genetical Theory of Natural Selection. He gave the theory a mathematical footing and brought broad scientific consensus that natural selection was the basic mechanism of evolution, thus founding the basis for population genetics and the modrn evolutionary synthesis, with Sewall Wright, which set the frame of reference for modern debates and refinements of the theory.

Generally speaking though, it seems a little unusual that the analysis of Big Tech and its history, as compared to the eventual scrutiny that Darwin's theories were given, seems slight. It is also interesting that there is little comment on where we are with Machine Learning and evolution.

We might also put Karl Marx (1818 - 1883) into the frame especially with reference to feudalism, the condition of the peasant, the serf and to think about the suggestion in the last section of Two Suns, Five, that we see the re-emergence of such conditions in neo feudalism or techno feudalism. It might be just too cute to think that techno feudalism burst out of a California garage in the 1990s. Again we might ask of Marx, what about the history of the machine 
before Bezos, in the nineteenth century and before? And we might ask of Marx, what about the liberation of the serf in this new context? What about data as capital?

As well as Marx and Darwin we might think of Freud who lived from 1856 to 1939 . To Freud the mind was something situated on two shoulders with a group of such to share. The idea of a mind operating in the screen of a phone with an onlooker, shoulders hunched perhaps, observing and occasionally contributing would have seemed preposterous in his time which takes us right up to the eve of the Second World War.

Coming back to before the First World War, in 1912 the world hears about Markov Chains. Andrey Markov first described techniques he used to analyse a poem. The techniques later become known as Markov chains. World War 1 (1914-18) saw the development of machines in the air and in communications. There is also a distraction as we know history because perhaps we look back to the causes of the war, who fought in it and who won the thing rather than the development of machines and science therein.

Turning to the general history of machines, the prehistory of machine learning, if you like, then we come, perhaps, to Alan Turing $(1912-1954)$ in a period of high curiosity driven, to some extent at least, by the Second World War (1939-45)and the development of the Enigma Machine, the breaking 
of codes and other things. In 1950 we have Turing's Learning Machine. Alan Turing proposes a 'learning machine' that could learn and become artificially intelligent. Turing's specific proposal foreshadows genetic algorithms.

After the Second World War we have an idea of brain, of mind. In 1951 there is the First Neural Network Machine. Marvin Minsky (1927-2016) and Dean Edmonds build the first neural network machine, able to learn, the SNARC. At the same time and with us still today we have the idea, the preoccupation perhaps, of and with machines that play games. In 1952 we have Machines Playing Checkers. Arthur Samuel joins IBM's Poughkeepsie Laboratory and begins working on some of the very first machine learning programs, first creating programs that play checkers.

1957 sees the Perceptron. Frank Rosenblatt invents the perceptron while working at the Cornell Aeronautical Laboratory. The invention of the perceptron generated a great deal of excitement and was widely covered in the media. The media coverage is important as in the next half century or so there are periodic flashes, occasional glimpses by the public of developments in AI.

Momentum continues into the 1960 s as Bayesian methods are introduced for probabilistic inference in machine learning. The emphasis on games continues. In 1963 we have Machines Playing TicTac-Toe Donald Michie creates a 'machine' 
consisting of 304 match boxes and beads, which uses reinforcement learning to play Tic-tac-toe (also known as noughts and crosses).

In 1964 Jack Ma, founder of Alibaba and Ant is born. His Wikipedia entry flies in the face of the idea that Big Tech leaders have illustrated genius in other areas like education:

...Ma struggled attending college. Ma failed the entrance exam for the Hangzhou Teachers College twice as his weak point was mathematics. The Chinese entrance exams, held annually, took Ma three years to pass. Ma attended Hangzhou Teacher's Institute... and graduated in 1988 with a Bachelor of Arts in English. While at school, Ma was head of the student council. After graduation, he became a lecturer in English and international trade at... Ma applied ten times to Harvard Business School and got rejected each time.

At the same time, staying with his Wikipedia entry, Ma shares with someone like Bill and Belinda Gates extreme wealth and a taste for philanthropy:

As of January 2021, with a net worth of $\$ 61.4$ billion, $\mathrm{Ma}$ is the third-wealthiest person in China, ...as well as one of the wealthiest people in the world, ranked 20th by Forbes. In 2019, Forbes named Ma in its list of "Asia's 2019 Heroes of Philanthropy" for his work supporting 
underprivileged communities in China, Africa, Australia, and the Middle East.

As shown later in this section, the history of Jack Ma in China is something of a work in progress. In 1967 we have Nearest Neighbor. The nearest neighbor algorithm was created, which is the start of basic pattern recognition. The algorithm was used to map routes. In some respects this looks ahead to the neighbourhood mesh discussed extensively in Seven of the Two Suns? series.

In 1968 2001: A Space Odyssey appears. This is an epic science fiction film produced and directed by Stanley Kubrick. The screenplay was written by Kubrick and Arthur C. Clarke, and was inspired by Clarke's 1951 short story The Sentinel and other short stories by Clarke. A novel released after the film's premiere was in part written concurrently with the screenplay. The film, which follows a voyage to Jupiter with the sentient computer HAL after the discovery of an alien monolith affecting human evolution, deals with themes of existentialism, human evolution, technology, artificial intelligence, and the possibility of extraterrestrial life. This film may be prescient in that Bezos and Musk are exploring both AI and space in 2021. What they will find and how this might affect human evolution are questions of the day. 
A year later in 1969 we have the Limitations of Neural Networks. Marvin Minsky and Seymour Papert publish their book Perceptrons, describing some of the limitations of perceptrons and neural networks. The interpretation that the book shows that neural networks are fundamentally limited is seen as a hindrance for research into neural networks.

But then in the 1970s, as noted above, there is what is known as the 'AI Winter' and a general pessimism about the effectiveness of machine learning.

Having said that there is a break in the weather so to speak, in 1970 when we have Automatic Differentiation (Backpropagation). Seppo Linnainmaa publishes the general method for automatic differentiation (AD) of discrete connected networks of nested differentiable functions. This corresponds to the modern version of backpropagation, but is not yet named as such.

1979 sees the Stanford Cart. Students at Stanford University develop a cart that can navigate and avoid obstacles in a room. Also in 1979 Kunihiko Fukushima first publishes his work on the neocognitron, a type of artificial neural network (ANN). Neocognition later inspires convolutional neural networks (CNNs).

The pendulum of interest swings back from the 'winter' of the 1970s in the 1980s as the rediscovery of backpropagation causes a resurgence in machine 
learning research. In 1981 Gerald Dejong introduces Explanation Based Learning, where a computer algorithm analyses data and creates a general rule it can follow and discard unimportant data. And in 1982 John Hopfield popularizes Hopfield networks, a type of recurrent neural network that can serve as content-addressable memory systems.

Then without a drum roll but with a redoubtable heft as time goes by, in 1983 there is the internet. Actually it is still a decade or so away but at the beginning of 1983, the Advanced Research Projects Agency Network (ARPANET) - a small network for academics and researchers - transitions to the standard TCP/IP protocol of the World Wide Web. The protocol would become the internet's cornerstone and technical foundation as it allows expanded available address space and decentralizes the network, thus also expanding accessibility.

In 1985 there is NetTalk. A program that learns to pronounce words the same way a baby does, is developed by Terry Sejnowski. This looks forwards in some ways to voice recognition which seems set to be the one of the next major developments in the 2020s. In 1986 there is a later version of backpropagation. Seppo Linnainmaa's reverse mode of automatic differentiation (first applied to neural networks by Paul Werbos) is used in experiments by David Rumelhart, Geoff Hinton and Ronald J. Williams to learn internal representations. Then in 
1989 Christopher Watkins develops Q-learning, which greatly improves the practicality and feasibility of reinforcement learning.

Also in 1989 there is the commercialization of Machine Learning on Personal Computers.

Axcelis, Inc. releases Evolver, the first software package to commercialise the use of genetic algorithms on personal computers. The appearance of Evolver might be 'the moment' in the evolution of the Algorithmic State, the point of tipping to the future where algorithms are ubiquitous, also a point, before the analysis of O'Neill and Sauter discussed in Five and elsewhere in the Two Suns? series, of, as it were, innocence, where the algorithm was simply seen as science and not as something that might be manipulated.

And the development of games continues to enrich this field of study. A few years later in 1992 we have machines playing backgammon. Gerald Tesauro develops TD-Gammon, a computer backgammon program that uses an artificial neural network trained using temporal-difference learning (hence the 'TD' in the name). TD-Gammon is able to rival, but not consistently surpass, the abilities of top human backgammon players.

Then in the 1990s work on machine learning shifts from a knowledge-driven approach to a data-driven approach. Scientists begin creating programs for computers to analyze large amounts of data and draw conclusions - or "learn" - from the results. 
Support vector machines (SVMs) and recurrent neural networks (RNNs) become popular. The fields of computational complexity via neural networks and super-Turing computation started. The shift to a data-driven approach might be another 'moment' in all this.

In 1994 Amazon.com is born in Seattle. With an initial aim of becoming an online bookstore, Jeff Bezos and a handful of angel investors launch Amazon.com, just as e-commerce is about to take off. This birth is not from an immaculate conception. It happens in a context of science which now has some force. The 'AI Winter' is over and, to really take liberties with metaphor, Amazon starts amidst a sparkling Spring of opportunities.

Also in 1994 Howard G Cunningham beigins coding the WikiWikiWeb and then he installs it on March 251995 as an add on to the Portland Pattern Repository.

Born in 1949 Cunningham is a 'Baby Boomer' like Bezos and the importance of Wikipedia which developed from his work and the input of others cannot be underestimated when one thinks, for example, of Cathy O'Neill's arguments about how data might be abused. Wikipedia stands out as a beacon nof truth in all this.

1995 sees the appearance of the Random Forest Algorithm. Tin Kam Ho publishes a paper describing random decision forests. Also in 1995 Corinna 
Cortes and Vladimir Vapnik publish their work on support vector machines.

Then there is another flash into public consciousness in 1997 when IBM Deep Blue Beats Kasparov at chess. Meanwhile, back in the lab, so to speak, in 1997 Sepp Hochreiter and Jürgen Schmidhuber invent long short-term memory (LSTM) recurrent neural networks, greatly improving the efficiency and practicality of recurrent neural networks.

How machines recognise things is important now as Facial Recognition, for example, becomes a major matter. In the next year, 1998, a team led by Yann LeCun releases the MNIST database, a dataset comprising a mix of handwritten digits from American Census Bureau employees and American high school students. The MNIST database has since become a benchmark for evaluating handwriting recognition.

In 1996 Robin Li develops a search engine called Rankdex which evolves into Baidu in 2000. According to Wikipedia:

Baidu is a Chinese multinational technology companyl specializing in Internet-related services and products and artificial intelligenced (AI), headquartered in Beijing's Haidian District. It is one of the largest $\mathrm{AI}$ and Internet companies in the world. The holding company of the group is incorporated in the Cayman Islands. 
The situation of Baidu in the Cayman Islands is significant in terms of the thesis offered in the Two Suns? series that the USA, possibly for reasons of security and defence has mostly been a 'home' for Big Tech. The discussion of Silicon Valley in California and techno feudalism in Five also applies here. The registration of a Chinese company originating in Beijing but eventually incorporating in a remote situation where the internet company possibly has more economic weight than the country it resides in resonates with the argument in earlier parts in this series where places like the Virgin Islands or Nauru were discussed as possible homes for the Algorithmic State. Of all the internet or Big Tech companies discussed Baidu best fits the model proposed in those early parts of this series. Also in 1998 what some call 'The Age of Google' begins in Menlo Park, California. As noted in USA Today (2020):

With seed money from Sun Microsystems cofounder Andy Bechtolsheim and Amazon founder Jeff Bezos, among others, Stanford University Ph.D. students Larry Page and Sergey Brin launch the search engine Google. The digital advertising behemoth Google Inc., now Alphabet Inc., is a $\$ 1.104$ trillion company with several subsidiaries, including video-sharing platform YouTube; autonomous-car development company Waymo; and $\mathrm{X}$, the company's research and development division. 
Also in 1998 Tencent is founded. It is telling to put
part of the Wikipedia entry alongside that of Google:

Tencent is the world's largest video game vendor, as well as one of the most financially valuable companies. It is among the larges social media, venture capital and investment corporations. Its services include social network, music, web portals, e-commerce, mobile games, internet services, payment systems, smartphones, and multiplayer online games. Offerings in China include the instant messengers, Tencent $Q Q$ and WeChat, and one of the largest web portals, QQ.com. It also owns the majority of Global's music services (Tencent Music Entertainment) with more than 700 million active users and 120 million paying subscribers.

The company surpassed a market value of US $\$ 500$ billion in 2018, becoming the first Asian technology company to cross this valuation mark. It has since then emerged as one of Asia's most valuable companies, and among the world's top technology companies by market value. Tencent has been credited as one of the world's most innovative companies by the Boston Consulting Group and Fast Company respectively. It has stakes in over 600 companies, began focusing on tech start-ups in Asia in 2017. TechCrunch characterized Tencent's investment strategy as letting its portfolio startups operate autonomously. 
Also in 1998 there is the founding of JD.com and again it is interesting to compare the Wikpedia entry with others:

JD.com, Inc., also known as Jingdong and formerly called 360buy, is a Chinese e-commerce company headquartered in Beijing. It is one of the two massive $\mathrm{B} 2 \mathrm{C}$ online retailers in China by transaction volume and revenue, a member of the Fortune Global 500 and a major competitor to Alibaba-run Tmall.

In 2000 the International Space Station Opens with low earth orbit about 250 miles above sea level. We might relate this to later developments like the shells of Amazon and its Kuiper satellites and Musk's Spacex operation or to the 2015 New Horizons expedition to the Kuiper Belt.

In 2002 we have the Torch Machine Learning Library. Torch, a software library for machine learning, is first released.

In 2004 Facebook is founded in Cambridge Massachusetts by Mark Zuckerberg then 23 years of age and others.

In 2006 The Netflix Prize competition is launched by Netflix. The aim of the competition was to use machine learning to beat Netflix's own recommendation software's accuracy in predicting a user's rating for a film given their ratings for 
previous films by at least $10 \%$. The prize was won in 2009.

In 2007 the iPhone is introduced in San Fransisco. As USA Today (2020) notes:

Apple CEO Steve Jobs (1955-2011) first shows the world one of the most popular branded consumer electronic devices in history, the iPhone. Since the first generation phone that Jobs introduced at the Consumer Electronics Show that year, there have been 18 versions of the mobile device, and more than 1.2 billion units have been sold globally through 2017. Only Samsung's Galaxy smartphone comes close to that volume.

Coming to 2009 and moving away from factual recounting on a year by year basis, to personal history and impressions the story of Tricia Wang about Nokia comes to mind:

...I've witnessed the struggle firsthand. In 2009, I started a research position with Nokia. And at the time, Nokia was one of the largest cell phone companies in the world, dominating emerging markets like China, Mexico and India -- all places where I had done a lot of research on how lowincome people use technology. And I spent a lot of extra time in China getting to know the informal economy. So I did things like working as a street vendor selling dumplings to construction workers. Or I did fieldwork, spending nights and days in internet cafés, hanging out with Chinese 
youth, so I could understand how they were using games and mobile phones and using it between moving from the rural areas to the cities.

Through all of this qualitative evidence that I was gathering, I was starting to see so clearly that a big change was about to happen among low-income Chinese people. Even though they were surrounded by advertisements for luxury products like fancy toilets -- who wouldn't want one? -- and apartments and cars, through my conversations with them, I found out that the ads the actually enticed them the most were the ones for iPhones, promising them this entry into this high-tech life. And even when I was living with them in urban slums like this one, I saw people investing over half of their monthly income into buying a phone, and increasingly, they were "shanzhai," which are affordable knock-offs of iPhones and other brands. They're very usable. Does the job.

The idea of participation in a smart world is incredibly strong and important in the notion of self. Tricia Wang also points out, in ways that correspond with the arguments throughout the Two Suns? series especially in the discussion of self in Seven, that modelling for humans on the basis of big data may be flawed:

And after years of living with migrants and working with them and just really doing everything that they were doing, I started piecing all these data points together -- from the things that seem random, like 
me selling dumplings, to the things that were more obvious, like tracking how much they were spending on their cell phone bills. And I was able to create this much more holistic picture of what was happening. And that's when I started to realize that even the poorest in China would want a smartphone, and that they would do almost anything to get their hands on one.

You have to keep in mind, iPhones had just come out, it was 2009, so this was, like, eight years ago, and Androids had just started looking like iPhones. And a lot of very smart and realistic people said, "Those smartphones -- that's just a fad. Who wants to carry around these heavy things where batteries drain quickly and they break every time you drop them?" But I had a lot of data, and I was very confident about my insights, so I was very excited to share them with Nokia.

But Nokia was not convinced, because it wasn't big data. They said, "We have millions of data points, and we don't see any indicators of anyone wanting to buy a smartphone, and your data set of 100 , as diverse as it is, is too weak for us to even take seriously." And I said, "Nokia, you're right. Of course you wouldn't see this, because you're sending out surveys assuming that people don't know what a smartphone is, so of course you're not going to get any data back about people wanting to buy a smartphone in two years. Your surveys, your methods have been designed to optimize an existing business model, and I'm looking at these emergent human dynamics that haven't happened yet. We're 
looking outside of market dynamics so that we can get ahead of it.

Well, you know what happened to Nokia? Their business fell off a cliff. This -- this is the cost of missing something. It was unfathomable.

Coming back to a year by year blow by blow analysis-and what a blow this was- in 2009 ImageNet was created. ImageNet is a large visual database envisioned by Fei-Fei Li from Stanford University, who realized that the best machine learning algorithms wouldn't work well if the data didn't reflect the real world. For many, ImageNet was the catalyst for the AI boom of the 21 st century and we might put it alongside Evolver as a tipping point in development.

With a head of steam, if you like, in AI Support Vector Clustering and other Kernel methods and unsupervised machine learning methods become widespread in the 2000s in sharp contrast to the flat period of the 1970s. Then in the 2010s deep learning becomes feasible, which leads to machine learning becoming integral to many widely used software services and applications. In 2010 Kaggle, a website that serves as a platform for machine learning competitions, is launched.

Then in 2010 the Wall Street Journal Profiles Machine Learning Investing. The WSJ profiles a new wave of investing and focuses on RebellionResearch.com 
which would be the subject of author Scott Patterson's novel, Dark Pools. The plot of this novel, as described in Goodreads is instructive:

A news-breaking account of the global stock market's subterranean battles, Dark Pools portrays the rise of the "bots"- artificially intelligent systems that execute trades in milliseconds and use the cover of darkness to out-maneuver the humans who've created them. In the beginning was Josh Levine, an idealistic programming genius who dreamed of wresting control of the market from the big exchanges that, again and again, gave the giant institutions an advantage over the little guy. Levine created a computerized trading hub named Island where small traders swapped stocks, and over time his invention morphed into a global electronic stock market that sent trillions in capital through a vast jungle of fiber-optic cables. By then, the market that Levine had sought to fix had turned upside down, birthing secretive exchanges called dark pools and a new species of trading machines that could think, and that seemed, ominously, to be slipping the control of their human masters. Dark Pools is the fascinating story of how global markets have been hijacked by trading robots-many so self-directed that humans can't predict what they'll do next. 
Throughout the Two Suns? series the transition from robotics to cobotics, from when humans made machines that were a little bit like them to the point where there was cooperation between human and robot to the further point where humans simply watched the machines as they worked at things humans used to do has been noted and the plot line of Scott Patterson's Dark Pools echoes that.

Shortly afterwards in 2011, using a combination of machine learning, natural language processing and information retrieval techniques, IBM's Watson beats two human champions in a Jeopardy! Competition. Again this takes all this into the public spotlight momentarily at least.

Then in 2012 The Google Brain team, led by Andrew $\mathrm{Ng}$ and Jeff Dean, create a neural network that learns to recognize cats by watching unlabelled images taken from frames of YouTube videos. In a Youtube clip, Peter Norvig, Director of Research at Google, demonstrates a computer system that has been programmed to organize collections of images from YouTube videos into sets of objects, without any direction from the programmers.

Also in 2012 as USA Today notes the "God Particle" is (probably) discovered in near Geneva.

Nearly 600 feet below the France-Switzerland border at CERN's Large Hadron Collider Facility, an international team of scientists discovers a new particle widely believed to be the elusive Higgs 
boson, known as the "God Particle," which is thought to be a fundamental component of the universe. Higgs boson has been an important element of particle physics theory for decades, but until 2012 there had been no physical evidence to support its existence.

2014 sees a leap in Face Recognition. Facebook researchers publish their work on DeepFace, a system that uses neural networks that identifies faces with $97.35 \%$ accuracy. The results are an improvement of more than $27 \%$ over previous systems and rivals human performance. Facial Recognition is considered several times across the Two Suns? Series with reference to its allowance in certain states and countries and its banning in others. DeepFace was an important step into all this.

Also in 2014, Sibyl Researchers from Google detail their work on Sibyl, a proprietary platform for massively parallel machine learning used internally by Google to make predictions about user behaviour and provide recommendations.

In 2015 NASA spacecraft New Horizons flies by Pluto some 3 billion miles from Earth and there is the inspiration, perhaps for Bezos' Kuiper satellites. As USA Today notes:

NASA becomes the first human-made object to fly past and observe the dwarf planet Pluto. New Horizons sends back stunning photographs of this 
enigmatic and distant member of the solar system, including images of a mountain range and massive icebergs floating in frozen nitrogen. New Horizons is now en route to the Kuiper Belt, a massive asteroid belt at the far reaches of the solar system.

Two years later in 2016 Google's AlphaGo program becomes the first Computer Go program to beat an unhandicapped professional human player using a combination of machine learning and tree search techniques. Later improved as AlphaGo Zero and then in 2017 generalized to Chess and more twoplayer games with AlphaZero.

Coming forward to 2016-7 we can see that the world is taken by surprise by the use of data in the Cambridge Analytica case. Molly Sauter sets the scene in 2017:

Earlier this year, a company called Cambridge Analytica shot to the forefront of the debate over big data and elections when it claimed responsibility for the upset victories of both Donald Trump and the Brexit Campaign. Reports have cast the firm as a puppet master "propaganda machine" able to mint voters through a proprietary blend of psychometric data, primarily Facebook "likes" and targeted nudges. In this story, repeated by Mother Jones and The Guardian among others, Cambridge Analytica [working in conjunction with an "election management" firm called SCL Group] is both a king maker and a Pied Piper: voters are unable to resist attempts at political manipulation, as they are seamlessly integrated with voters' online 
environment and pulled by strings too deeply anchored in voters' psyches to be ignored.

Like Cathy O'Neill Molly Sauter has some key phrases such as 'the techno-magic of underdescribed big data psychographics' and 'algorithmic nudging':

I'm uninterested in the actual snake content of Cambridge Analytica's snake oil. As noted by the MIT Technology Review and BuzzFeed, the company has made some big claims and has been happy to take credit for several of 2016's startling electoral results. But Cambridge Analytica relies heavily on the techno-magic of under-described big data psychographics and algorithmic nudging. Both the Tech Review and BuzzFeed point out that the amount and types of data that the company appears to use are not much different than types of data acquisition and analysis already commonly in use.

As Molly Sauter continues a phrase that stands out is 'data objects':

Instead I'm interested in the ways that Cambridge Analytica's sales pitch reflects how the subjects of these big data analytics projects are viewed by those conducting the research, and the entitlements held by advertisers, tech firms, and researchers who deploy big data analytics in support of political campaigns or other political 
projects. This sense of entitlement matters. I'd like to posit that the use of "big data" in politics strips its targets of subjectivity, turning individuals into ready-to-read "data objects," and making it easier for those in positions of power to justify aggressive manipulation and invasive inference. I would like to further suggest that when big data methodology is used in the public sphere, it is reasonable for these "data objects" to, in turn, use tactics like obfuscation, up to the point of actively sabotaging the efficacy of the methodology in general, to resist attempts to be read, known, and manipulated.

Molly Sauter's argument looks forward to the discussion in Seven of the Two Suns? series on data doppelgangers as the nature of the self in the context of Big Tech is considered. Tricia Wang's experiential discussion above comes to mind as well here.

In a talk of 2017 on Youtube Professor Andrew Ng declares again that 'AI is the new electricity'. Andrew $\mathrm{Ng}$ has moved from the Google Brains Team and the business of computers recognising cats discussed earlier in 2012 having also been the former chief scientist at Baidu, where he led the company's Artificial Intelligence Group and he thinks that just as electricity transformed every major industry so will AI especially in regard to 'deep learning'. The comparison or analogy is significant in terms of the thread of this part of the Two Suns? series in that it shows a worthwhile effort to organise the mindset 
about AI just as it shows the difficulty perceived in explaining it. Andrew Ng is, as we say, an important guy in all this.

In 2020, after Amazon expands from books to the so-called "Everything Store" and growing a business selling cloud services to companies like Netflix and Instagram, Geoff Bezos becomes the world's richest man. At this time there is something of a standoff between Bezos, the owner of the Washington Post and Donald Trump the President of the United States. This is unusual as there is not a lot of noise between Big Tech and Big Government in the normal course of things. In fact there is a good deal of 'smoothing' as we see such things as, say, Belinda Gates talking about this important thing to do for the planet or that in short clips regularly on CNN.

But the Trump-Bezos standoff may be a shade of the full colour seen in a fascinating and important example of the relation between Big Tech and the nation state of China where Jack Ma was 'away' for three months in 2021. According to Sam Peach of BBC Radio Documentaries of March 20, 2021:

The Alibaba founder had accused Chinese banks of operating with a "pawn-shop mentality". He had also claimed that the authorities were trying to "use the way to manage a railway station to manage an airport" when it came to regulating the new world of digital finance. 
These statements angered the banking establishment and reportedly reached the attention of President Xi Jinping.

If we make the above sequence of events more central to our history rather than obscure then we might come to general background for what is happening with the development of the algorithmic state.

Just as the 1970s are sometimes referred to as an 'AI winter' we might think of generally inclement intellectual conditions for the consideration of the machine until we get to Wells (1866 - 1946) when 'the Shakespeare of Science Fiction' brings his writing to the world.

The idea of an AI winter is interesting in some respects and may be worth looking at the Wikipedia definition before proceeding:

In the history of artificial intelligence, an AI winter is a period of reduced funding and interest in artificial intelligence research.The term was coined by analogy to the idea of a nuclear winter.The field has experienced several hype cycles, followed by disappointment and criticism, followed by funding cuts, followed by renewed interest years or decades later.

The term first appeared in 1984 as the topic of a public debate at the annual meeting of AAAI(then called the "American Association of Artificial Intelligence"). It is a chain reaction that begins with 
pessimism in the AI community, followed by pessimism in the press, followed by a severe cutback in funding, followed by the end of serious research. At the meeting, Roger Shank and Marvin Minsky-two leading AI researchers who had survived the "winter" of the 1970s-warned the business community that enthusiasm for AI had spiraled out of control in the 1980s and that disappointment would certainly follow. Three years later, the billion-dollar AI industry began to collapse.

Hype is common in many emerging technologies, such as the railway mania or the dot-com bubble. The AI winter was a result of such hype, due to over-inflated promises by developers, unnaturally high expectations from end-users, and extensive promotion in the media Despite the rise and fall of AI's reputation, it has continued to develop new and successful technologies. AI researcher Rodney Brooks would complain in 2002 that "there's this stupid myth out there that AI has failed, but AI is around you every second of the day. In 2005 Ray Kurzweil, agreed: "Many observers still think that the AI winter was the end of the story and that nothing since has come of the AI field. Yet today many thousands of AI applications are deeply embedded in the infrastructure of every industry.

Enthusiasm and optimism about AI has generally increased since its low point in the early 1990s. Beginning about 2012, interest in artificial intelligence (and especially the sub-field of machine learning) from the research and corporate 
communities led to a dramatic increase in funding and investment.

Is the story of an AI winter a cautionary tale for Big Tech? The position taken throughout the Two Suns? Sseries is that its too late now for another 'winter' just as it is for ignorance or denial, the cat is out of the bag and established in the hood. Part of this position is the recognition that we now face something of a stand alone culture when we consider Big Tech and its use of data, algorithms, machine learning and the like. The term 'algorithmic state' has been used by the Milan theorists, a set of commentators linked to Bocconi University. We might return to the point that the political anthropology, so far at least, would appear to admit no structures beyond the nation state in its terms of reference. The Milan theorists seem to tie developments using algorithms to the law of the nation state. Here the position taken is that the Algorithmic State has now got a life of its own. In the Wikipedia quote above possibly the most important points are made by Rodney Books and Ray Kurzweil that the use of AI is ubiquitous, omnipresent and embedded. Again, to push a couple of metaphors, we may have been looking at a silent Spring without noticing the flowering of its political and social effects.

For another walk down or along a socio-legal path we might turn again to the recent Sackur-Lina Khan 
interview on Hardtalk in early 2021. This is introduced on the BBC website as follows:

Can and should anything be done to halt the inexorable rise of the global technology giants such as Amazon, Google and Facebook? Over the past decade we've seen these tech titans come to dominate data collection, cloud computing, retail, social media and publishing, but now there is pushback from anti-monopoly lawyers and sceptical politicians. Stephen Sackur speaks to the American lawyer Lina Khan, who is at the forefront of the movement to tame big tech.

But whose interest is she serving?

Sackur starts by talking about Lina Khan's background, her age of 31, her coming from England to get interested in journalism and the law especially law pertaining to big tech. Notable here was the antitrust paradox article discussed at length by me in the first Two Suns series especially in One and Two. The paradox would appear to be that while Amazon appears to offer better service at a better rate the competition is stifled and stymied. Interestingly, Sackur does not mention the Chicago School or the idea that so long as the price is affordable by the public the product is alright and nor does Khan throughout the interview.

Also notable is that both Sackur and Khan, like the Milan theorists never seem to get past the nation state as the beginning and end of analysis. As 
discussed earlier events in Myanmar and elsewhere indicatre a different reality.

The interview starts with Khan, lead by Sackur, asserting that there is a significant momentum to 'tame big tech.

Khan speaks of a change in public opinion and a wave of lawsuits. Sackur speaks of increased power by big tech in the time of lockdown. Khan responds by saying yes and these firms 'provide infrastructure', 'these forms control the core infrastructure for commerce and communications'. There is a small group pf executives setting the rules. the infrastructure heretofore according to Khan had been bound or accountable to public rules and regulations.

Sackur asks whether the services provided by big tech are good or not. Khan says even if these services are good people and institutions may be hurt and she asks what are we missing out on. Also monopoly power can inhibit competition.

After inquiring how Khan got there Sackur is told by Khan that when working as a journalist Khan found that across sectors control was falling into fewer hands.

This lead to problems as consumers, as workers and as citizens. A systemic process across the US economy. On the one hand there were monopoly laws but there had been an 'ideological transformation'. Sackur speaks of a simple notion of price and as long as the customer is served with the 
right price for a reasonable product all was well. They both agree that there is more to it than price. Khan uses the analogy of a King with too much power being like a corporation with too much power this being the reason for anti monopoly laws.

Sackur then turns to the way people like Amazon offer low cost and efficiency. Khan questions Sackur's idea of efficiency saying that operational efficiency is one thing but market dominance is another. Khan also suggests that the price situation is difficult to assess because of fluctuations. They argue about Amazon, Sackur suggesting that there is competition and Khan giving statistics for Amazon dominance in the US.

The exploitation of third parties is discussed by Khan saying that they cannot compete effectively. Sackur, going back to Brandeis, says this is just the way of bigness. Khan then talks about fair practices and suggests that big tech is predatory rather than competing on merit. Also these firms are infrastuctory, if that is a word and a different set of rules apply to infrastructure, big tech offering essential infrastructure. Like the railways there is a gatekeeper role being taken and this requires additional scrutiny and regulation.

Sackur asks of the US government is ready for a bis fight with big tech. He refers to Khan's earlier work withn the Federal Trade Commission. Khan talks about action in this area and talks about 'landmark cases'. Sackur asks how hard the government will 
press this. He asks whether Tim Wu's idea of needing to break up the companies as the only option will occur. Facebook should relinquish What's Up and other acquisitions.

Khan talks of ways to break up Big Tech including legislation and lawsuits.

Sackur moves to Biden and his appointment of Renata Hesse and tech apologists in Biden's top tier. Khan notes concerns in this area. Then Sackur refers to Hesse's comments on Google and the fact that people like it. Khan shifts the argument to the UK where Google's dominance is said to inhibit competition. Then says that Hesse's view is out of date and not corresponding to reality.

Sackur asks why European states are harder on big tech. He talks about Margaret Vestager and asks about financial clout of big Tech in the US. Khan says big tech puts money into jurisdictions in the US and the Europeans are 'clear eyed' about big tech.

The question is what remedy works and now Europeans are looking to stronger remedies. They do not talk about the standoff between the Australian government and Facebook.

Sackur turns to companies as 'content providers. He gives the example of Trump being taken off Twitter and the shut down of Parler because it lost its Amazon server. He speaks of the 'unaccountable powers' of big tech. Khan says its quite coherent to think both of these things, that Trump should have 
been removed and that its not OK for power to be in so few hands This is a problem for democracy.

Sackur mentions Angela Merkel's opposition to the Twitter move and says these firms are serving as core communications infrastructure.

Sackur suggests that this is a matter of the firms being treated as publishers under US law which they never have been in the past. Khan talks about Section 230 which exempts these firms from liability.

Khan suggests that the business models of these companies encourages hate speech or the like and that model might be questioned. Finally, Sackur asks whether these firms will be bigger or what in ten years time. Khan says yes and refers to the first gilded age which is discussed in Three of the Two Suns? series.

The Sackur-Khan discussion is important in terms of the history considered in this section as it shows what the scope of discourse is now in 2021.The discussion opened doorways in a profitable way into such things as infrastructure. Are we talking about the infrastructure of nation state or the infrastructure of a parallel cyber or algorithmic state?

\section{Conclusion}

To take up one of the main threads in this Two Suns? series, that there are several spaces or forms of space involved; territorial space, outer space, 
cyberspace and living space, and there is data to be found and owned in each of these spaces while that data may be processed using algorithms in each space and across all spaces, each kind of space has a history. And the way data and algorithms are used in each space and across them has a history as well. That history might be about, as Cathy O'Neil suggests, the data and algorithms involved in the recipe for baking a pie or the planning of a space station.

With such points in mind it has been suggested in this section that a mind shift is required to a new sense of culture and history. The machine, the learning machine could well be central in such a consciousness. A history of AI, robotics and learning machines has been offered via Wikipedia to suggest something of a central thread.

The way people grapple with this history if they do so at all is usually with reference to the nation state. Understandable as this might be in the analysis of the Sackur/Khan interview and elsewhere it is suggested that such a framework is limiting and somewhat of a distraction.

Going back to the discussion in Two Suns? Five we might see this history in terms of the development of Big Tech and think again about Kotkin's thesis of 2020 that California is moving into techno feudalism. We might also consider the rise and development of the internet and its centrality in our 
history in order to understand, Facebook, Netflix and other Big Tech operators as well as understanding ourselves, the spaces we live in and the way our identities are formed in this context. The next section of the Two Suns? series considers this. 


\section{Bibliography}

Bayes Thomas, 'An Essay towards solving a Problem in the Doctrine of Chances' (ed. Price, Richard and communicated to John Canton) Philosophical Transactions of the Royal Society of London. 53: 370-418 1763

Cleave, Peter Ka hao te kupenga hiko, He Tuhinga Aronui: the Journal of Maori Writing ed. Moon, P AUT 2001

Dick, Phillip K Do Androids Dream of Electric Sheep? Doubleday 1968

Foucault, Michel The Order of Things Routledge 1970

Hart Rob The Warehouse Bantam 2019

Kotkin, Joel 'Neo-Feudalism in California' American Affairs Volume IV, Number 2 Summer 2020

Laplace, Pierre-Simon Théorie Analytique des Probabilités Wentworth Press 2018

Mannheim, Karl Ideology and Utopia Martino Fine 2015

Minsky, Marvin and Papert, Seymour (2nd edition with corrections, first edition 1969) Perceptrons: An Introduction to Computational Geometry, The MIT Press 1972

O'Neill, Cathy Weapons of Math Destruction, Crown 2016

Patterson, Scott, Dark Pools: The Rise of the Machine Traders and the Rigging of the US Stock Market Crown 2013 
Peach, Sam of BBC Radio Documentaries of March 20, 2021,

Sauter, Molly Persuasion and the other thing: A critique of big data methodologies in politics in ETHNOGRAPHY MATTERS, Facebook 2017 Wells H G The Time Machine Heinemann 1895 Wood, Gaby Living Dolls, A Magical History of the Quest for Mechanical Life, Faber and Faber 2002

\section{Internet sources}

Ng, Andrew 2017 Artificial Intelligence is the new electricity

https://www.youtube.com/watch?v=21EiKfQYZXc Giridharadas, Anand (2019) Winners Take All Video RSA Minimate

2010 the Wall Street Journal Profiles Machine Learning Investing.

Goodreads entry on Dark Pools

Youtube on Norvig 2017 on Youtube Professor Andrew Ng https://www.youtube.com/watch?v=VasJplgi3z8 Tricia Wang: The human insights missing from big data $\mid$ TED ...

https://www.usatoday.com/story/money/2020/0 9/06/the-worlds-most- important-event-everyyear-since-1920/113604790/

Norvig Youtube clip on object recognition https://www.youtube.com/watch?v=TK4qLwTye_s Sackur-Lina Khan interview on Hardtalk 2021 
Tricia Wang: The human insights missing from big \begin{tabular}{l|l} 
data & TED Talk
\end{tabular} 